\title{
INFLUENCE OF DENTURE PALATAL CONTOURING ON THE OROPHARYNGEAL SPACE IN COMPLETE DENTURE WEARERS: A CROSS-OVER RADIOGRAPHIC STUDY
}

\author{
Elsayed A. Abdel-Khalek*, Abdullah M. Ibrahim* and Wael M. Aboelmaaty**
}

\begin{abstract}
Objectives: The aim of this 2- years cross-over study was to radiographically compare the influence of palatal contouring of maxillary denture on oro-pharyngeal airway space.

Methods: The study was conducted on 20 healthy patients who were completely edentulous in the maxilla and mandible. All patients were randomly allocated in two equal groups to receive a mandibular denture opposed by a maxillary denture with either an arbitrary contoured palate (ACP) or a functional contoured palate (FCP) at the start of the study. The maxillary denture was exchanged after 1 year from insertion. The oropharyngeal volume (OPV) and minimum cross sectional area (CSA) were measured by using CBCT.
\end{abstract}

Results: After 12 months of denture insertion, the mean OPV was more with FCP $\left(29.9 \pm 3.1 \mathrm{~cm}^{3}\right)$ compared to ACP denture $\left(24.9 \pm 2.2 \mathrm{~cm}^{3}\right)$, also the mean CSA was more with FCP denture $\left(363.7 \pm 67.3 \mathrm{~mm}^{2}\right)$ compared to ACP denture $\left(286.5 \pm 65.8 \mathrm{~mm}^{2}\right)$. There was a high positive correlation between OPV and CSA with FCP denture $(r=0.90)$ and ACP denture $(r=0.61)$.

Conclusion: Within this limited study, fabricating a complete denture with functionally contoured palatal surface may have a positive role in maintaining the oropharyngeal airway patency especially in old patients.

\section{INTRODUCTION}

Edentulous patients with prominent anatomical changes are subjected to muscular discomfort with altered phonetic and masticatory functions that may be functional in nature, namely, muscular hypotonicity ${ }^{(1,2)}$.
Loss of occlusal vertical dimension with posterior mandibular rotation affects the hyoid position and reduces the pharyngeal airway space ${ }^{(3)}$. In addition, the genioglossus muscle that is the pharyngeal dilator muscle, has an important anatomical location and function in reducing the upper airway patency ${ }^{(4)}$. Such reduction in

* Lecturer of Removable Prosthodontics, Faculty of Dentistry, Mansoura University, Egypt.

** Lecturer of Oral and Maxillofacial Radiology, Faculty of Dentistry, Mansoura University, Egypt. 
pharyngeal space may be reversible by complete denture insertion ${ }^{(5)}$. Functionally, the genioglossus activity is well maintained in normal individuals during wakefulness as well as while asleep ${ }^{(4)}$.

Complete denture prosthesis restores an acceptable degree of masticatory function, improves phonetics, and provides esthetics by restoring both adequate support to orofacial musculatures and proper vertical dimension of the face ${ }^{(6)}$. Additionally, wearing denture induces modifications in the position of the tongue and favors the patency of upper airway space especially in patients with sleep disorders ${ }^{(7)}$.

Indeed, insertion of an intraoral prosthesis would have an indirect effect on the tongue, soft palate, and the patency of the airway ${ }^{(8,9,10)}$. The study of Gagnon et al. ${ }^{(11)}$ on dentate patients, advocated that a bite elevation without a protrusive component might due to the risk of aggravating the upper airway obstruction for some patients. In this concern, Nayar and Knox ${ }^{(12)}$ reported that complete dentures fabricated with an acceptable occlusal vertical dimension might not change the horizontal mandibular position in denture wearers.

Emami et al. (2) found that edentulous elderly with poor sleep quality suffered from low oral health quality of life independent of nocturnal prosthesis wearing. Impaired function of the genioglossus muscles and pharyngeal inflammation was reported also with dentures wearing ${ }^{(6,13)}$. Literatures stated that both the lingual flange and palatal plate are responsible for the encroachment of the tongue and reduction in upper airway volume ${ }^{(10,14)}$. Furthermore, the shape of the palatal contour may affect the functions of the tongue and soft palate that will alter the pharyngeal airway volume ${ }^{(7)}$.

According to previous studies, the change in pharyngeal airway space may be a result of concentric collapse of the hypopharynx or collapsed lateral oropharyngeal walls, tongue, and soft palate against the posterior wall of the pharynx ${ }^{(5,15)}$.
Cephalometric radiography method that generates two-dimensional images may not valid for assessment of three-dimensional structures ${ }^{(16)}$. Therefore, several studies assessed the airway volume by using 3D segmentation of Cone Beam Computed Tomography $(\mathrm{CBCT})$ images ${ }^{(16,17,18)}$.

There was a limited data regarding the influence of palatal contouring of complete denture on these measurements in patients free from sleep disorders. The aim of this 2-years study was to compare the influence of palatal contouring of maxillary denture on oro-pharyngeal space volume (OPV) and minimal cross sectional area (CSA) using CBCT in normal healthy complete denture wearers. The null hypothesis of this study was that there is no significant difference in the oro-pharyngeal airway space regardless the palatal contour of the denture.

\section{MATERIALS AND METHODS}

\section{Patients Selection:}

The study was conducted on 20 healthy patients (14 men and 6 women) who were completely edentulous in the maxilla and mandible. The selected patients had an age range of 46- 65 years (mean 56 years). The participants presented to Department of Removable Prosthodontics, Faculty of Dentistry, Mansoura University for complete denture treatment. At least 6 months of being completely edentulous patient was eligible criteria for the selection in the study, with no previous experience of denture use. Subjects with a history of temporomandibular disorders, major cardiorespiratory problems, sleep disorders, head/neck injury, or with skeletal deformity were excluded. All participants had Angel's class I maxilla-mandibular relation with 20 to $30 \mathrm{~mm}$ inter-arch space without severe undercuts in their residual alveolar ridges.

For all participants, a screening visit was performed starting with intraoral clinical examination then body's height, weight, and neck 
circumference were also recorded, and then their body-mass index (BMI) was calculated (Appendix 1). They were asked about their sleep history and were required to complete self-administered the Epworth Sleepiness Scale (ESS) questionnaires (Appendix 2) to assess their sleepiness during daytime (scores ranging from 0 to 24). Subjects who have scores $\geq 10$ indicate sleepiness and they were excluded according to Johns' method ${ }^{(19)}$. Informed consent was written from each patient, and approval from the Faculty Ethical Committee was obtained.

\section{Prosthetic Procedures:}

The following procedures were performed for each patient as follows:

1. Preliminary impressions were made by using irreversible hydrocolloid impression material (Cavex CA37, Holland), which were poured for the fabrication of diagnostic plaster cast. Custom trays were fabricated using autopolymerizing acrylic resin (Acrostone, cold cure, Egypt). Border molding was perfomed with low fusing impression compound sticks (DPI Pinnacle Tracing Sticks, India) and final impressions were made with zinc oxide eugenol-free impression material (Cavex outline, Holland). Final impressions were poured into master casts by using hard dental stone (Durguix, Hard natural stone, Spain).

2. The maxillary master cast was duplicated for each patient, then maxillary and mandibular record blocks were constructed, and the contours were adjusted regarding the height and orientation relation. The maxillary face bow transfer was used for mounting the maxillary master cast on the articulator (Whip Mix Corporation, USA).

3. Vertical and horizontal relations were recorded by using interocclusal wax wafer method. A centric interocclusal record was used for mounting the mandibular master cast. The articular was adjusted for eccentric movements by the protrusive record then bilateral balanced occlusion was developed.

4. After setting the acrylic artificial teeth, waxing up the dentures was performed with arbitrary contouring the palatal surface. The second set of trial maxillary dentures was obtained by using negative silicone molds with soft wax pouring method according to Teraoka et al ${ }^{(20)}$. The trial dentures were processed into long-cycle heat cured acrylic resin (Acrostone, denture base material, Egypt), and then the dentures were finished and polished.

5. After processing, the second set of processed maxillary dentures were modified by removing the palatal area (Fig. 1A) and replacing it by functionally contoured palatal surface (FPC) that was constructed by swallowing technique with tissue conditioning material (Lynal tissue conditioner Dentsply, USA) (Fig. 1B ) according to Lott and Levin (21), and Shaffer and Kutz (22). The modified denture with FPC was converted into auto-polymerized acrylic resin (Palapress Vario Heraeus Kulzer, Germany) then was placed in a pressure pot for 20 minutes.

6. All patients were randomly allocated in two equal groups considering the gender (with7 men and 3 women in each group) to receive a mandibular denture opposed by a maxillary denture with either arbitrary contoured palate (ACP) or functional contoured palate (FCP) at the start of the study.

\section{II- CBCT Scanning Protocol and Assessment Method:}

CBCT was performed using a cranial X-ray CT scanner (i-CAT Vision ${ }^{\circledR}$, Imaging Sciences International, Hatfield, PA, USA). The CBCT scanning protocol was as follows: $90 \mathrm{kVp}, 5 \mathrm{~mA}, 14$ $\mathrm{x} 10 \mathrm{~cm}$ field of view, $280 \mu \mathrm{m}$ voxel and a scanning time of $16 \mathrm{~s}$. 
The patients of both groups (FCP or ACP start) had to wear the dentures for a 2-weeks adaptation period (T0). After the adaptation period (baseline), they underwent $\mathrm{CBCT}$ imaging for measuring the OPV and minimum CSA. According to Furuya et al ${ }^{(6)}$, CBCT exposures were taken for the subject in upright position with the natural position of the head and maximal intercuspal position of the teeth at the end of expiration. To ensure reproducibility as possible, patient-scanner distance and the height of the stretcher were recorded in the first scan.

The projections in the sagittal, coronal and axial orthogonal planes were primary reconstructed and then the images of the entire volume of craniofacial complex were stored in DICOM format
(Digital Imaging Communications in Medicine). Oropharynx was outlined superiorly through the horizontal plane extending from the posterior nasal spine (PNS). Inferiorly, the area was outlined by the tip of epiglottis opposite to most infero-anterior point of the third cervical vertebra (C3).

The oro-pharyngeal area posterior to the tongue base and the soft palate was semiautomatically segmented the OPV volume in $\mathrm{cm}^{3}$ of A threedimensional model with the software (Fig. 2A). The cross-sectional area (CSA) between the tongue base "tip of epiglottis" and pharyngeal wall was located and measured in $\mathrm{mm}^{2}$ (Fig.2B)

After 12-month period, patients underwent a second CBCT scanning (T12) to record the start

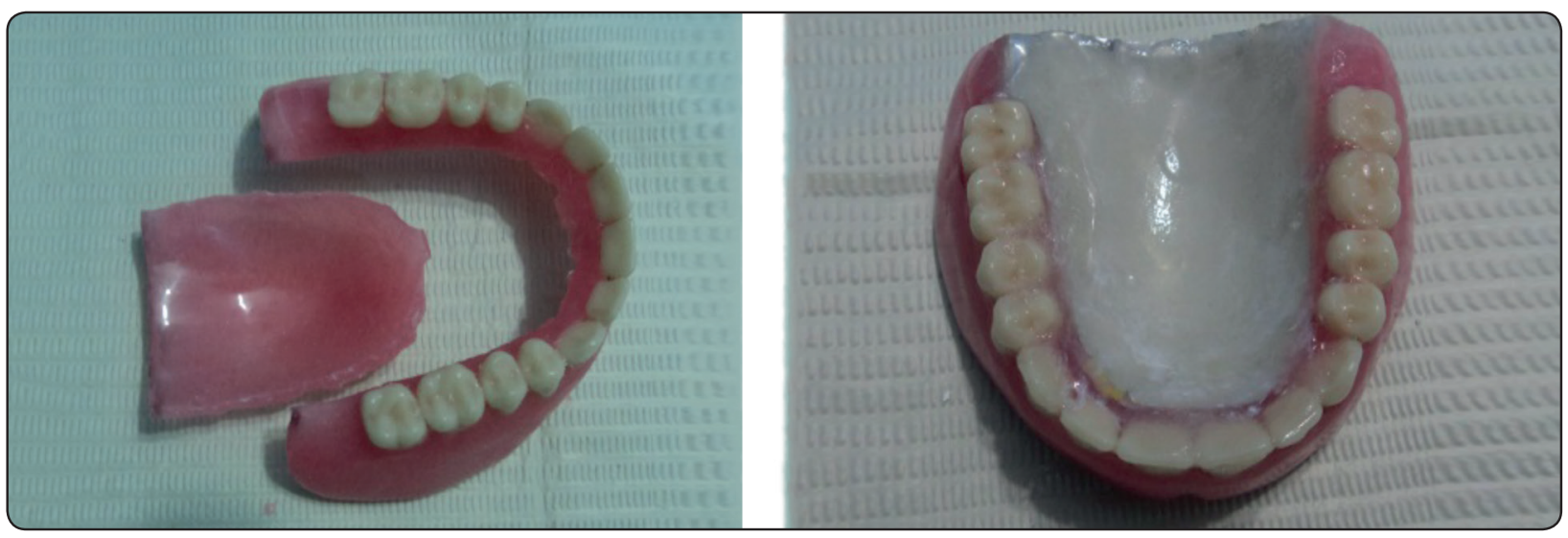

Fig. (1) A processed maxillary denture was modified by removing the palatal area (A) image on the left, and functionally contoured by using tissue conditioning material (B) image on the right.

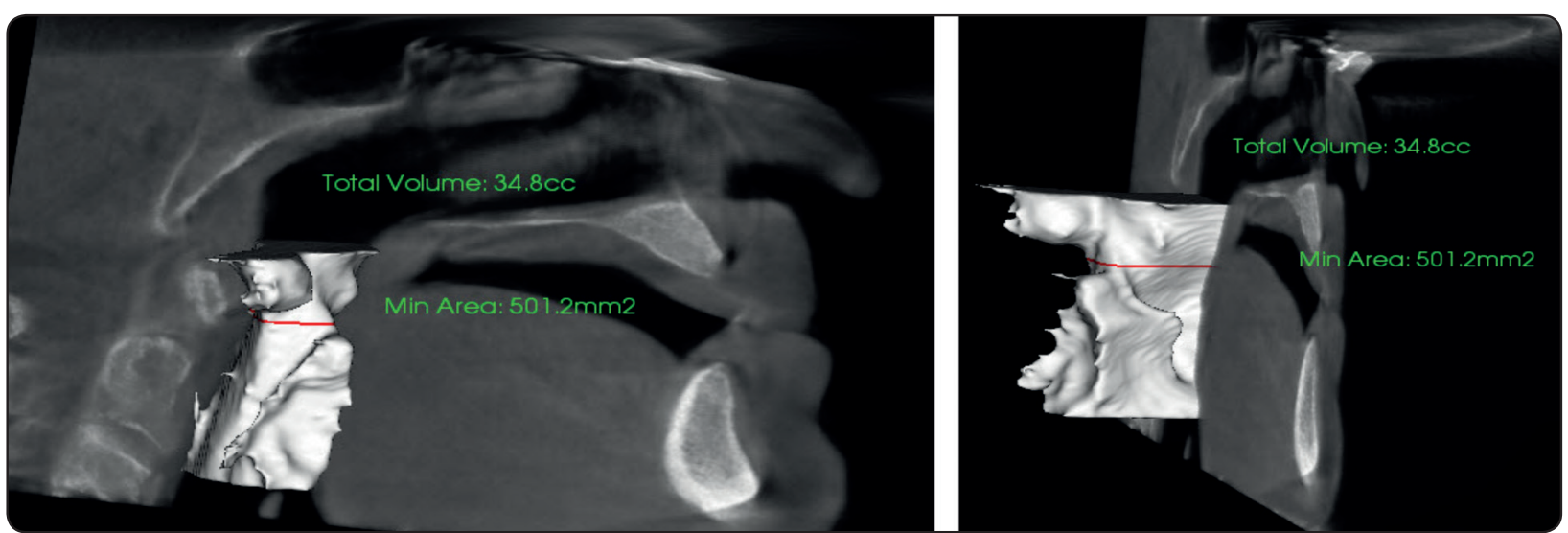

Fig. (2) CBCT of 3D segmented oropharynx with measured volume and minimal cross-sectional area saggital section on the left (A)., coronal section on the right (B). 
denture, and then the maxillary dentures were exchanged and the occlusion was adjusted accordingly. The measurements of a third CBCT scanning were repeated after another 12-month to record the latter denture. Therefore, each denture group has two measurements (To and T12). At the end of the study, the patients had the option to keep the maxillary denture of their choice for continuous use.

The collected data were analyzed using SPSS v 17.0 (SPSS Inc., Chicago, IL). A parametric evaluation plan was used after checking the recorded data for normality by using (KolmogorovSmirnov tests) to test normal distribution. Paired $\mathrm{t}$ test was used to compare between ACP denture $(n=20)$ and FCP denture $(n=20) . P<0.05$ indicated a statistically significant association. The OPV and CSA were compared by linear regression analysis using a correlation coefficient (r).

\section{RESULTS}

The minimum CSA and OPV, for ACP dentures and FCP dentures are shown in Tables 1. Following 2-weeks from denture insertion (T0), The mean OPV was $\left(23.3 \pm 2.1 \mathrm{~cm}^{3}\right)$ with FCP denture compared to $\left(23.2 \pm 1.7 \mathrm{~cm}^{3}\right)$ with ACP denture, also the mean minimum CSA was $\left(295.5 \pm 70.2 \mathrm{~mm}^{2}\right)$ with FCP denture compared to $\left(281.5 \pm 69.8 \mathrm{~mm}^{2}\right)$ with ACP denture.

At T12, the mean OPV was more with FCP $\left(29.9 \pm 3.1 \mathrm{~cm}^{3}\right)$ compared to $\left(24.9 \pm 2.2 \mathrm{~cm}^{3}\right)$ with ACP denture, also the mean minimum CSA was more with FCP denture $\left(363.7 \pm 67.3 \mathrm{~mm}^{2}\right)$ compared to $\left(286.5 \pm 65.8 \mathrm{~mm}^{2}\right)$ with ACP denture.
At the base line (T0), there was a statistically insignificant difference between FCP group and ACP group when compared regarding minimum CSA ( $p=0.08)$. While, comparing the significance at $\mathrm{T} 12$, there was a statistically significant increase of CSA with FCP denture group compared to ACP ( $p=0.002$ ) as shown in Table 1. Similarly, on comparing OPV for FCP against ACP at T0, there was a statistically non-significant differences ( $p$ $=0.06$ ). While after 12 months (T12), there was a statistically significant increase of OPV with FCP denture group in comparison to $\mathrm{ACP}(\mathrm{p}=0.04)$.

For minimum CSA, comparing the significance within each denture group between $\mathrm{T} 0$ against $\mathrm{T} 12$ revealed a statistically high significant differences for FCP denture as well as ACP denture $(\mathrm{p}<0.001)$. Similar results were obtained for OPV , there was a statistically high significant differences for FCP denture as well as ACP denture group $(\mathrm{p}<0.001)$ as presented in Table 1.

A linear regression was used to model the relationship between volume and area for both ACP and FCP groups at T12 (Table 2 \& Fig. 3). A best-fit line was found on a scatter plot of OPV and CSA. The $\mathrm{R}^{2}$ value was 0.37 and 0.82 for ACP denture and FCP denture, respectively. The ACP denture group demonstrated a weaker (less linear) relation between OPV and CSA. There was a high correlation between the minimum CSA and OPV with FCP denture $(r=0.90)$ and ACP denture $(r=$ 0.61 ). The oropharynx with the smallest CSA had the smallest OPV.

TABLE (1) Comparison of minimal cross section area (CSA in $\mathrm{mm}^{2}$ ) and oropharyngeal volume (OPV in $\mathrm{cm}^{3}$ ) measurements at baseline (T0), after 12-month period (T12) in ACP and FCP dentures.

\begin{tabular}{|c|c|c|c|c|c|c|}
\hline & \multicolumn{3}{|c|}{ Minimal CSA $\left(\mathbf{m m}^{\mathbf{2}}\right)$} & \multicolumn{3}{c|}{ OPV $\left(\mathbf{c m}^{3}\right)$} \\
\hline & $\begin{array}{c}\text { T0 } \\
\text { Mean } \pm \text { SD }\end{array}$ & $\begin{array}{c}\text { T12 } \\
\text { Mean } \pm \text { SD }\end{array}$ & P-value & $\begin{array}{c}\text { T0 } \\
\text { Mean } \pm \text { SD }\end{array}$ & $\begin{array}{c}\text { T12 } \\
\text { Mean } \pm \text { SD }\end{array}$ & P-value \\
\hline ACP $(\boldsymbol{n}=\mathbf{2 0})$ & $269.3 \pm 64.8$ & $286.5 \pm 65.8$ & $<0.001$ & $23.2 \pm 1.7$ & $24.9 \pm 2.2$ & $<0.001$ \\
\hline FCP $(\boldsymbol{n}=\mathbf{2 0})$ & $295.5 \pm 70.2$ & $363.7 \pm 67.3$ & $<0.001$ & $23.3 \pm 2.1$ & $29.9 \pm 3.1$ & $<0.001$ \\
\hline P-value & 0.08 & 0.002 & & 0.06 & 0.04 & \\
\hline
\end{tabular}

t test,

highly significant $\quad P<0.001$.

Significant $P<0.05$ 
TABLE (2) Correlation between minimal cross section area (CSA) and oropharyngeal volume (OPV) measurements after 12-months for all patients $(n=20)$ in the study .

\begin{tabular}{|c|c|c|c|c|}
\hline \multirow{2}{*}{} & \multicolumn{2}{|c|}{ FCP denture } & \multicolumn{2}{c|}{ ACP denture } \\
\cline { 2 - 5 } & \multicolumn{2}{|c|}{ OPV } & \multicolumn{2}{c|}{ OPV } \\
\hline & $\mathrm{r}$ & P-value & $\mathrm{r}$ & P-value \\
\hline CSA & 0.90 & $<0.001$ & 0.61 & 0.02 \\
\hline
\end{tabular}

r: Pearson Correlation, $\quad$ highly significant $P<0.001$. Significant $P<0.05$

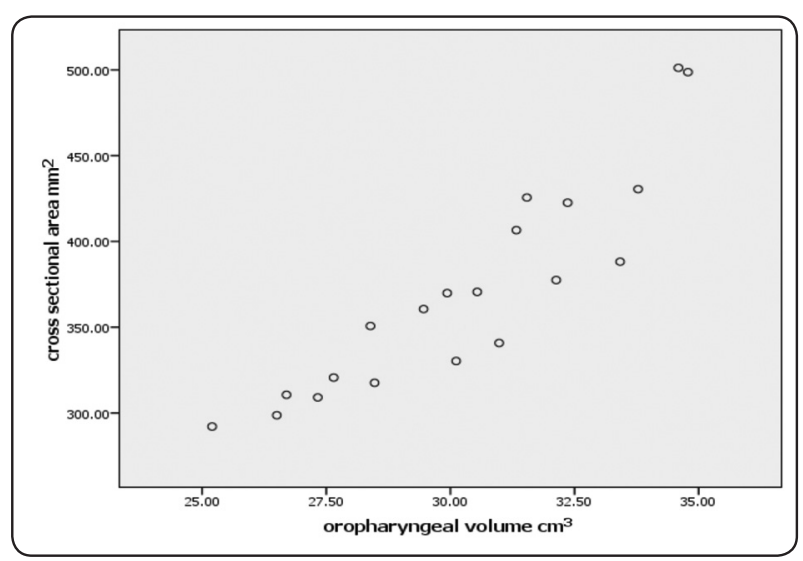

Fig. (3) Graph depicting strong positive correlation (R2=0.82) between volume and minimal cross-sectional area in FCP denture group.

\section{DISCUSSION}

Complete edentulism might be one of possible factors that contributing to increased pharyngeal collapsibility because of impaired function of muscles dilating the upper airway or pharyngeal inflammation from denture wearing ${ }^{(2,3,6,15)}$. Complete denture not only improves esthetics, mastication, and phonetics but also provide better support to orofacial musculature by restoring proper vertical dimension of the face. However, literatures stated that insertion of the intraoral prosthesis causes deflection of the tongue that may encroach the airway space ${ }^{(9)}$. The literatures confirmed that wearing dentures induces modifications in the tongue, soft palate, position of the jaw, and consequently pharyngeal airway space changes ${ }^{(7)}$.
In accordance with Padmanabhan, et al (23) who stated that dimensional change in soft tissues surrounding the airway space in complete denture wearers might be reversible. To avoid influence of confounding factors, each patient in the current study design was allowed to be a control for himself.

The current study considered the confounding factors that could qualitatively and quantitatively affect the size and shape of the oropharyngeal airway at the time of image acquisition. To preclude the confounding risk factors for sleep disorders, confirmatory questionnaires were performed to exclude obstructive sleep apnoea OSA. Obesity and increased neck circumference were reported as important risk factors for OSA in the general population especially in older adults age 60 and over (24). The patients were screened for BMI and increased neck circumference. BMI is an inexpensive, and easy-use method for population assessment of overweight and obesity because the calculation requires only height and weight ${ }^{(15,25,26)}$. ESS (range 0-24) was used to measure the level of perceived daytime sleepiness, and scores $\geq 10$ indicate sleepiness subsequently, those patients were excluded from the study ${ }^{(19)}$.

Despite a validated and optimized CBCT protocol for airway imaging remains elusive, it has been commonly used to assess the effect of various orthodontic and surgical treatments with reliable anatomical linear and volumetric measurements ${ }^{(27)}$. Moreover, it was reported that minimum CSA area is a crucial measure of flow limitation and airway collapsibility with excellent intra-examiner and inter-examiner reliability ${ }^{(6,17)}$.

According to a systematic review conducted by Gurani et al ${ }^{(28)}$ both airway volume and CSA showed an increase when evaluated in respect to head extension, head rotation, altered body position, and jaw protrusion. Therefore, the radiographic technique was standardized during CBCT scan in this study. The patient was seated in upright position 
and was instructed to avoid swallowing or any other movements during the exposure.

The mandible was maintained in a reproducible position with teeth in maximal interdigitation and occluding in centric relation. In addition, the hyoid position was standardized in this study by taking the exposures at the end of expiration. In line with this precaution, Guijarro-Martinez and Swennen ${ }^{(27)}$ reported that respiratory phase and tongue posture were variables for upper airway imaging technique.

The results of this study observed a change in CSA and OPV after 12-month from denture insertion. These findings are in accordance with previous studies that demonstrated significant changes in retropharyngeal space with wearing of complete dentures fabricated with acceptable vertical dimension of occlusion ${ }^{(2,3,6,23,26)}$.

The study recorded a mean of OPV $29.9 \pm 3.1$ $\mathrm{cm}^{3}$ and $24.9 \pm 2.2 \mathrm{~cm}^{3}$ with minimal CSA area $363.7 \pm 67.3 \mathrm{~mm}^{2}$ and $286.5 \pm 65.8 \mathrm{~mm}^{2}$ for FCP and ACP groups, respectively. The minimal CSA was located between the tongue base and pharyngeal wall. This observation concurred with Ogawa et al. ${ }^{(29)}$ who reported that the areas posterior to the soft palate and the base of the tongue are recognized as the narrowest in the upper airway's total space. It was observed that the mean minimum CSA measured in previous studies, conducted on normal individuals, was narrower than the current study ${ }^{(29,30)}$. The results reported in the present study may be attributed to the age-related pharyngeal expansion that increases the pharyngeal dimensions in older participants compared to the young age participants in the previous studies ${ }^{(6)}$. Another reason for data variations between studies is that measurements could be affected by the body position during CBCT airway examination. This explanation concurred with Camacho et al ${ }^{(31)}$ who found that the airway was smaller when patients were in a supine compared with an upright position.

The observed high positive correlation between OPV and minimum CSA with FCP denture ( $r=$ 0.90 ) could be logic as the increase in the CSA is associated with increase in the total pharyngeal volume similar to findings of Furuya et al ${ }^{(6)}$. These findings may be attributed to the faster tongue adaptation by the activity of the genioglossus muscle. The associated anterior movement of the tongue's base and epiglottis may result in increased horizontal expansion of the oropharynx ${ }^{(6,32)}$.

This study included some limitations; firstly, static method of CBCT scan in an upright patient may not mimic dynamic motion of the soft tissues and hyoid bone that control the change in size and shape of the upper airway. Secondly, the oropharyngeal dimension may be varied significantly between males and females; however, this was not reported in the current study because of limited sample size and few number of female participation.

\section{CONCLUSION}

Within the limitation of the present study, it could be concluded that;

- Well-designed complete denture prosthesis and regular follow-up care may reduce the hazardous effect on dimensional changes of the surrounding pharyngeal tissues.

- Fabricating a complete denture with functionally contoured palatal surface may have a role in maintaining patency of the upper airway in older patients.

- Further investigations using longitudinal studies may be useful in determining the relationship between complete denture design and the upper airway patency. 


\section{APPENDIX 1}

\section{Questionnaire For The Completely Edentulous Subjects}

\section{PERSONAL DETAILS:}

- Participant number: e.g. 001

- Sex:

- Date of Birth:

- Height (cm):

- Weight (Kg):

- BMI (Weight/[Height $\left.]^{2}\right)$ :

- Neck Circumference $(\mathrm{cm})$ :

\section{MEDICAL/DENTAL HISTORY}

- Medication:

- Allergies:

- Cardiac problems (high BP, angina, heart attack):

- Respiratory problems (asthma, emphysema, bronchitis):

- Other problems (DM, TMD, thyroid, head/neck injury):

- Do you snore? (Yes/No)

- Has your partner/family ever complained that you snore? (Yes/No)

- Do you have a good night's sleep? (Yes/No)

- Do you often wake up feeling tired? (Yes/No)

- What is the date of last extracted tooth? (where?)

- Do you currently use a removable denture? (Yes/No)

\section{INTRA-ORAL EXAMINATION}

- Angel's maxilla-mandibular relation:

- Available inter-arch space of mounted diagnostic casts (mm):

- Ridge undercuts (None/moderate/severe):

\section{APPENDIX 2}

\section{Epworth Sleepiness Scale (Ess):}

How likely are you to doze off or fall asleep in the situations described below? Use the following scale to choose the most appropriate number for each situation:

0:would never doze. 1:light chance of dozing.

2:moderate chance of dozing. 3: high chance of dozing.

\begin{tabular}{|l|l|l|l|l|}
\hline \multicolumn{1}{|c|}{ SITUATION } & \multicolumn{3}{c|}{ CHANCE OF } \\
DOZING
\end{tabular}

Total score ( $\geq 10$ indicate excessive daytime sleepiness): 


\section{REFERENCES}

1. Bucca C, Cicolin A, Brussino L, Arienti A, Graziano A, Erovigni F, et al. Tooth loss and obstructive sleep apnoea. Respir Res. 2006;7:8.

2. Emami E, Salah, MH, Rompre, P, Huynh, N, Beauchamp, A, Feine, JS. The nocturnal use of complete dentures and sleep stability in edentulous elders. J Dent 2013;41:703-9.

3. Douglass JB, Meader L, Kaplan A., Ellinger CW. Cephalometric evaluation of the changes in patients wearing complete dentures: A 20-year study. J Prosthet Dent 1993;69:270-5.

4. Mezzanotte WS, Tangel DJ, White DP. Waking Genioglossal Electromyogram in Sleep Apnea Patients versus Normal Controls (a Neuromuscular Compensatory Mechanism). J Clin Invest. 1992;89:1571-9.

5. Gokce SM, Akin E, Bengi O. Effects of complete denture wearing on the head posture and posterior airway space: A cephalometric study. J Dent Sci 2011;6:6-13.

6. Furuya J, Tamada Y, Sato T, Hara A, Nomura T, Kobayashi $\mathrm{T}$. Wearing complete dentures is associated with changes in the three-dimensional shape of the oropharynx in edentulous older people that affect swallowing. Gerodontology 2015; 33: 513-21.

7. Erovigni F, Graziano A, Ceruti P, Gassino G, De Lillo A, Carossa S.. Cephalometric evaluation of the upper airway in patients with complete dentures. Minerva Stomatol $2005 ; 54: 293-301$.

8. Cleall JF. Circumstances limiting the development and verification of a comprehensive theory of craniofacial morphogenesis. Acta Morphol Neerl Scand 1972; 10:115-26.

9. Brown EC, Cheng S, McKenzie DK, Butler JE, Gandevia SC, Bilston LE. Tongue and lateral upper airway movement with mandibular advancement. Sleep 2013;36:397-404.

10. Nikolopoulou M, Ahlberg J, Visscher CM, Hamburger HL, Naeije M, Lobbezoo F,. Effects of Occlusal Stabilization Splints on Obstructive Sleep Apnea: A Randomized Controlled Trial. J OrOfac Pain 2013;27: 199-205.

11. Gagnon Y, Mayer P, Morisson f, rompré PH, Lavigne GJ. aggravation of respiratory disturbances by the use of an occlusal splint in apneic patients: a pilot study. Int $\mathrm{J}$ Prosthodont 2004;17:447-53.
12. Nayar S. and Knox J. Management of obstructive sleep apnea in an edentulous patient with a mandibular advancement splint: a clinical report. J Prosthet Dent. 2005;94:108-11.

13. Emami E, Lavigne G, de Grandmont P, Rompré PH, Feine JS. Perceived sleep quality among edentulous elders. Gerodontology 2012; 29: e128-e34.

14. Ozbek MM, Miyamoto K, Lowe AA, Fleetham JA. Natural head posture, upper airway morphology and obstructive sleep apnoea severity in adults. Eur J Orthod 1998;20:133-43.

15. Johal A, Patel S, Battagel JM.. The relationship between craniofacial anatomy and obstructive sleep apnoea: a casecontrolled study. J Sleep Res. 2007; 16, 319-26.

16. Burkhard JPM, Dietrich AD, Jacobsen C, Roos M, Lubbers HT, Obwegeser JA. Cephalometric and three-dimensional assessment of the posterior airway space and imaging software reliability analysis before and after orthognathic surgery. J Cranio-Maxillofacial Surg. 2014;42:1428-36.

17. Chen H, Aarab G, de Ruiter MHT, de Lange J, Lobbezoo F, vander Stelt PF. Three-dimensional imaging of the upper airway anatomy in obstructive sleep apnea: A systematic review. Sleep Med. 2016;21:19-27.

18. Zimmerman JN, Lee J, Pliska BT. Reliability of upper pharyngeal airway assessment using dental CBCT: a systematic review. Eur J Orthod. 2016:cjw079. doi:10.1093/ejo/cjw079.

19. Johns MW.Anew method for measuring daytime sleepiness: the Epworth sleepiness scale. Sleep 1991;14:540-5.

20. Teraoka F, Nakagawa M, Takahashi J. Adaptation of acrylic dentures reinforced with metal wire. J Oral Rehabil 2001; 28:937-42.

21. Lott F. and Levin B. : Flange technique anatomic and physiologic approach to increase retention, function, comfort, and appearance of dentures. $\mathrm{J}$ prosth Dent. 1966; 16:394-413,.

22. Shaffer FW and Kutz, RA. Phonetic and swallowing to determine palatal contours of dentures. J prosth Dent. 1972;28: 360-2.

23. Padmanabhan TV, Banu RF, Mahalakshmi A, Aziz A, Bohra S, Kumar VA.. Dimensional change in soft tissues with complete dental prosthesis and its effect on airway space and natural head position. Indian J Dent Res. 2015; 26:144-7. 
24. Davies RJ, Stradling JR. The relationship between neck circumference, radiographic pharyngeal anatomy, and the obstructive sleep apnoea syndrome. Eur Respir J. 1990;3:509-14.

25. StrobelRJ, Rosen RC. Obesity and weight loss in obstructive sleep apnea: a critical review. Sleep 1996;19:104-15.

26. Chaccur DC, Bittencourt LRA, 'Lucchesi L, Almeida FR, Frigerio MLMA. Assessment of the impact of vertical dimension alterations on the quality of sleep in elderly patients wearing upper and lower full dentures. Sleep Sci. 2012;5:1-6

27. Guijarro-Martínez R, Swennen GRJ. Three-dimensional cone beam computed tomography definition of the anatomical subregions of the upper airway: A validation study. Int J Oral Maxillofac Surg. 2013;42:1140-9.

28. Gurani SF, Di Carlo G, Cattaneo PM, Thorn JJ, Pinholt EM. Effect of Head and Tongue Posture on the Pharyngeal Airway Dimensions and Morphology in
Three-Dimensional Imaging: a Systematic Review. J oral Maxillofac Res. 2016;7:1-12.

29. Ogawa T, Enciso R, Shintaku WH, Clark GT. Evaluation of cross-section airway configuration of obstructive sleep apnea. oral Surg oral Med oral Pathol oral Radiol Endod, 2007; 103: 102-8.

30. Smith, J.M. The normal adult airway in 3-dimensions: A cone-beam computed tomography evaluation establishing normative values. MSc Thesis, University of Michigan (2009).

31. Camacho M, Capasso R, Schendel S. Airway changes in obstructive sleep apnoea patients associated with a supine versus an upright position examined using cone beam computed tomography. J Laryngol Otol. 2014;128:82430 .

32. Cheng CF, Peng CL, Chiou HY, and Tsai CY. Dentofacial morphology and tongue function during swallowing. Am J. Orthod Dentofacial Orthop. 2002;122:491-9. 